\title{
People, Practices, and Patterns: Transforming into a Learning Institution
}

\author{
Bill Heinrich, Breana Yaklin, Dave Goodrich, and Jess Knott
}

\begin{abstract}
Organizational transformation takes time, energy, and patience. An active administrative unit, The Hub for Innovation in Learning and Technology (called simply, the Hub) engaged intentional change from its inception. The Hub aspires to help Michigan State University (MSU) "reinvent itself as a learning institution” by transforming ourselves so that we might transform others. The authors, all current employees of the Hub, provide an in-depth look into the challenges and triumphs of purposeful organizational change. Without change, MSU would maintain existing gaps on measures of student success among different demographic groups. The key audience of these efforts are learners in the university while considering the surrounding system of faculty, staff, administrators, curriculum, assessment, and student engagement efforts. The Hub catalyzes innovative ways to collaborate, learn, research, and improve learning. We design new opportunities that take advantage of skills and connections across the disciplines. The Hub adapted work practices that can sustain transformation. The Hub also built a physical space reflective of these strategies. Initial practices resulted in a series of careful, bold learning pathways focused on new capacity and professional development for stakeholders. We extrapolated experiences, practices, and findings for other transforming university, business, and organizational contexts.
\end{abstract}

Keywords: organizations; systems change; spatial development; self-reflection; capacity

\section{Introduction}

Existing cultural practices and paradigms can yield difficult habits to break, even when those practices no longer serve students well or provide any other benefit (Kuhn, 1962). A key response to a desire or need for change includes making a number of organizational, structural, relational, symbolic, or staffing changes (Bolman \& Deal, 2013). Unfortunately, the strategies employed by leaders and faculty either lack coordination or simply address problems at the surface without addressing underlying causes (Senge, 2006). Within this article, the authors will discuss the evolution of an academic-administrative unit at Michigan State University (MSU), and how this organization, the Hub for Innovation in Learning and Technology (the Hub) seeks to reinvent MSU as a learning institution.

With the transformations the Hub has begun to implement, MSU intends to address organizational learning from multiple perspectives simultaneously. To accomplish perspective taking, a key practice of the Hub is regular self-reflection to learn from experiences. The Hub also engages its partners in regular self-reflection to create solutions that encompass the underlying complexity of persistent problems. 


\section{Value Proposition}

In early 2015, a unique set of leaders, ideas, and budgets coalesced into a committed network of faculty and staff focused on curriculum, technology, assessment, and culture building. These factors combined to create the Hub. In just a few months, MSU chartered this organization and charged it with "reinventing MSU as a learning organization," (Grabill, J., personal communication, 2015). As a result, the Hub sought to provide a focus for the university in addressing the following related opportunities:

- The opportunity to enhance MSU's pedagogical and technological support for learning while building human capacity for the continuation of that support.

- The opportunity to identify high-value learning outcomes and ways to research, measure, and analyze those outcomes. In the domain of research on learning analytics, for example, MSU began and helped lead a conversation about what inclusive and highquality learning looks like in higher education and is working to realize that opportunity.

- The opportunity to do innovative work in the domain of undergraduate and graduate education.

The Hub also sought to address a number of new challenges:

- The lack of a clear and focused intellectual and physical space on campus concerned with the identification, facilitation, and development of scalable learning innovations that can aid large numbers of students.

- The lack of a space on campus that could serve as an incubator for new learning models, new uses of space, new uses of technology, and new forms of collaboration.

- The lack of a strategy for online and digital learning that encompasses both on-campus and distance learning. Related to this is the necessity to execute that strategy in line with the mission of the university.

As a support entity, the Hub aimed to facilitate better learning pathways through pedagogy, technology, accessibility, and connections between learners and teachers. The Hub intended to find out what students need and want out of their educational experience. The potential value to students at MSU became clearer through a series of ongoing leadership conversations, with purposeful student involvement. These conversations included creating a space for active, engaged, and influential learning and working to close the gap between faculty and students by increasing communication and eliminating barriers. But how?

To approach these aims, the Hub modeled the behaviors it wanted to see in the university. An early task of the Hub was to generate creativity and urgency for interventions in student academic success. In this effort to create organizational change, the Hub began with people the staff believed to be interested in improving student outcomes. This included not only team members, but project partners as well.

At the same time, the staff had to build its own team. Coming together from several different administrative units on campus, Hub staff had to build rapport internally and begin to create a 
non-hierarchical organizational practice, characterized as a "flat" culture. This meant that the Hub staff collectively expressed feedback for projects and processes daily. As part of the staff, we were asked to challenge assumptions and establish new processes to do so in a way that created conversation, rather than prescription. Staff also had to be welcomed to offer feedback and apply their skills in a way that would help evolve the collaborative culture with both our team members and our project partners. As the Hub learned and evolved, a story emerged which detailed the journey the Hub took toward progress.

To tell this story, we first ground our discussion in the literature of learning organizations. Next, we focus on how people enact their practices and patterns as examples of a transformative organizing principle. Finally, we ${ }^{1}$ describe how a nimble organization emerged over time as a means to reinvent MSU as a learning institution by targeting student learning experiences and outcomes (Figure 1).

\section{TIMELINE SPECTRUM OF THE HUB}

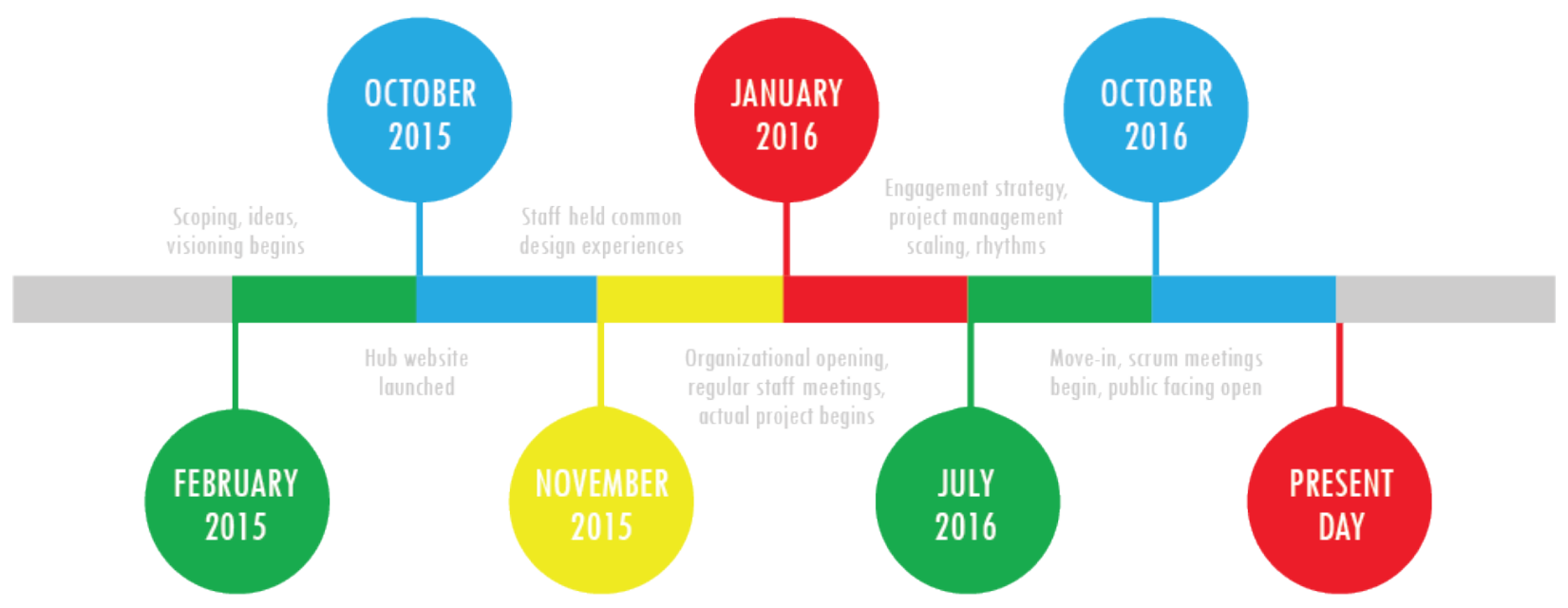

Figure 1. A timeline and practice spectrum of the Hub.

\section{Review of Relevant Literature}

\section{Transformational Learning}

The development of the Hub organization has been an experience in transformation. According to Mezirow, "a defining condition of being human is that we have to understand the meaning of our experience," (1997, p. 5). The development of Hub work and culture focuses on this idea, and has become a frame of reference for staff. Further, we have focused this guiding principle

${ }^{1}$ Authors wish to thank the Hub for time and support to write this manuscript, Emma Kukuk for editorial guidance and Keesa Johnson and Momoko Rai for insights on early drafts. 
outward in an attempt to understand how our campus partners are experiencing this change with us. According to Mezirow:

“A frame of reference encompasses cognitive, conative, and emotional components, and is composed of two dimensions: habits of mind and a point of view. Habits of mind are broad, abstract, orienting, habitual ways of thinking, feeling, and acting influenced by assumptions that constitute a set of codes. These codes may be cultural, social, educational, economic, political, or psychological. Habits of mind become articulated in a specific point of view - the constellation of belief, value judgment, attitude, and feeling that shapes a particular interpretation,” (1997, p. 5-6).

Intentional reflection among staff and project partners is a key component of transformative learning, and purposefully informs each stage of project development, as well as into expectations for staff. Continuous learning and experimentation is the customary practice, as is sharing what was learned from failures in the process. As found in Liimatinen, Poskiparta, Karhila et al. (2001), reaching the level of critical learning requires a focus on the practice of intentional reflection as a part of the learning process. In addition to learners, Hub staff are also educators, requiring another layer of critical reflection. Cranton \& Carusetta (2004) recommend that those in teaching roles reflect upon their sense of self as not only teachers but also people, finding that those who do are more likely to articulate the values their practice is based upon and share it with others.

Kreber (2004) noted that while reflection is important, and many people say they build opportunities for reflection into their work, this is not often the case, as those same people frequently cannot provide an account of their activity that demonstrates engagement in reflection. We counteract this tendency by operationalizing intentional reflection as an activity. We also use reflection as a means of documenting and sharing practices.

Transformational learning has many meanings ranging from micro changes in neural connections to wholly changed individual identities and choices. We have identified a set of observations and outcomes about our own organizational changes. Changes in individual habits, physical spaces and uses, and social interactions across the organization receive our attention. We also share stories and evidence of our adopted goal of transforming ourselves in order to transform the larger MSU institution.

\section{Being a Learning Organization}

Organizations that learn from data and experiences can transform. The essential process is similar to individual transformation, although it requires more conscious thought and coordination in a group or organizational setting. The Hub emerged within this complex and mature higher education environment to provide important symbolic, cultural, and technical support for solving problems in student-success issues. Without coordinating efforts to address complex issues and problems, stakeholders likely perceive that we say one thing but do another. We say we value student graduation rates but may appear to sideline this goal when the institution does not address the issue with sufficient resources (Argyris \& Schön, 1996). In this environment, addressing multifaceted academic issues require a clear vision, unified efforts, and 
strong leadership. Understanding this environment guides our interactions with stakeholders who have differing interests and motivations, sufficient political will to change organizational patterns, and resources to make measurable progress. Without intentional coordination to address student success issues in universities, individuals and colleagues with different perspectives may unintentionally oppose each other in order to protect their local interests.

In contrast, a learning organization works to recognize tensions between competing perspectives in an effort to create solutions or interventions that value feedback from different stakeholders (Senge, 1996). A catalyst and a result of these practices is a shared understanding or meaning of the purpose of the work. These kinds of organizations move quickly and slowly at the same time. They work fast to build partnerships and create prototypes, while working deliberately to peel back the layers of an issue and examine the connections and feedback loops of the underlying system.

In many ways, the Hub's organizational transformation effort relies on an understanding of systems thinking. Systems thinking relies on people to "make their understanding of social systems explicit” for the purposes of improvement (Aronson, 1996, p 1.). For example, gaps in student success across identity markers is typically a symptom of larger societal challenges. Systems thinking encourages addressing complex issues by trying to address underlying problems. Previous approaches to student success have included additional student support for different groups of students based on identities, representing symptom-based approaches. The Hub works to approach this problem systemically as a means to encourage enrollment management, student support, financial aid, and academic departments to work together. Systems thinking requires us to address the underlying causes and the environment in which the problem occurs. According to Aronson, systems thinking is helpful when facing:

“...complex problems that involve helping many actors see the 'big picture' and not just their part of it; recurring problems or those that have been made worse by past attempts to fix them; issues where an action affects (or is affected by) the environment surrounding the issue, either the natural environment or the competitive environment; [and] problems whose solutions are not obvious” (1996, p. 1).

Contrary to linear problem analysis, systems thinking seeks not to take apart the elements of an argument, but rather to look for the ways that those parts and their movements affect one another and observe how short-term decisions affect long-range consequences.

The Hub aims to employ systems thinking by creating shared meaning among stakeholders and facilitating opportunities for organizational reflection. By learning as an organization, we expect that our group might experience a transformation. The object of this transformation is simultaneously the Hub itself and the larger entity of MSU. We adopt Senge's (2006) description of “learning organizations” as:

“...those organizations where people continually expand their capacity to create the results they truly desire, where new and expansive patterns of thinking are nurtured, where collective aspiration is set free, and where people are continually learning to see the whole together," (p. 4). 
The Hub strives to be a learning organization. The staff and leaders encourage and reward both self and group reflection. In essence, we reflect the sentiment that a learning organization is a group of people working together collectively to enhance their capacities to create results of value for many people (Fulmer \& Keys, 1998). These documented reflections serve to guide communication among the Hub team, our project partners, and institutional stakeholders.

From Ideas to Action: The First Project of the Hub is the Hub

The Hub has aligned its intentions and activities in order to reinvent MSU as a learning institution. We further this goal by a few patterns and behaviors that, taken individually, might not make an impact. However, taken together, when integrated into a work system, these patterns and behaviors have the potential for extraordinary impact throughout the organization of MSU. Activities the Hub uses are Design Thinking, scheduled and unscheduled (pop-up) coworking, regular brainstorming, weekly coordinated staffing rhythms, project staffing decisions, and selfreflective behaviors, all of which we will explain herein. Planning our behaviors and work patterns was the first step towards achieving our goal of creating an innovative, open, and collaborative workspace. The next step was to commit to these behaviors in our daily work and follow through on our intentions of diffusing these behaviors across our team and throughout campus.

At its core, the Hub is a design and consulting operation working on behalf individual and teams of stakeholders at MSU. Staff at the Hub listen for opportunities and charter projects. A charter describes appropriate people, time, and resources deployed on behalf of faculty and department led change efforts. Projects have ranged from course level teacher professional development, to department wide curriculum and assessment practices, to institution wide analytics and decision support. Because the Hub receives funds from the campus provost (general funds) no charges are made for services. However, the project charters specify the ways in which the Hub staff will work closely with the project partner. At the end of projects, the project partner 'owns' the solution and the Hub does not maintain support beyond that point.

\section{Intentional Transformation}

Design thinking. We began to use Design Thinking as a guiding framework for both our internal processes and to organize our projects from our first conversations. The Hub uses two definitions of design thinking to guide its work. At its core, design thinking is a method of "of meeting people's needs and desires in a technologically feasible and strategically viable way," (Brown, 2008, para. 2). According to Turnali (2013), it is also “...a process, applicable to all walks of life, of creating new and innovative ideas and solving problems,” (para. 3). Design thinking methods play an important role in our ongoing work as the Hub in that they allow us to systematically assemble and make sense of disparate views and conflicting campus needs in order to collaborate on and create applicable solutions. We have begun to collect evidence of the impact on project goals and learning gains made by project partners because of working with design thinking approaches. We value the way design thinking encourages teams to work through conversations. At the same time, we lack robust evidence to make claims about success with these approaches. 
We consider our use of design thinking as an ongoing inquiry with periodic benchmarks to help us evaluate the efficacy.

Coworking. Coworking is one of the keys to our work philosophy, and one of the ways we facilitate and execute the ideas, solutions, and innovations identified in our design thinking work. Coworking events bring stakeholders campus-wide into a shared space where they can work on their own projects, while sharing ideas and contributing to projects as desired.

"Culture cups" (Figure 2) are used to illustrate availability, and set workspace norms for focus and personal work time and space.

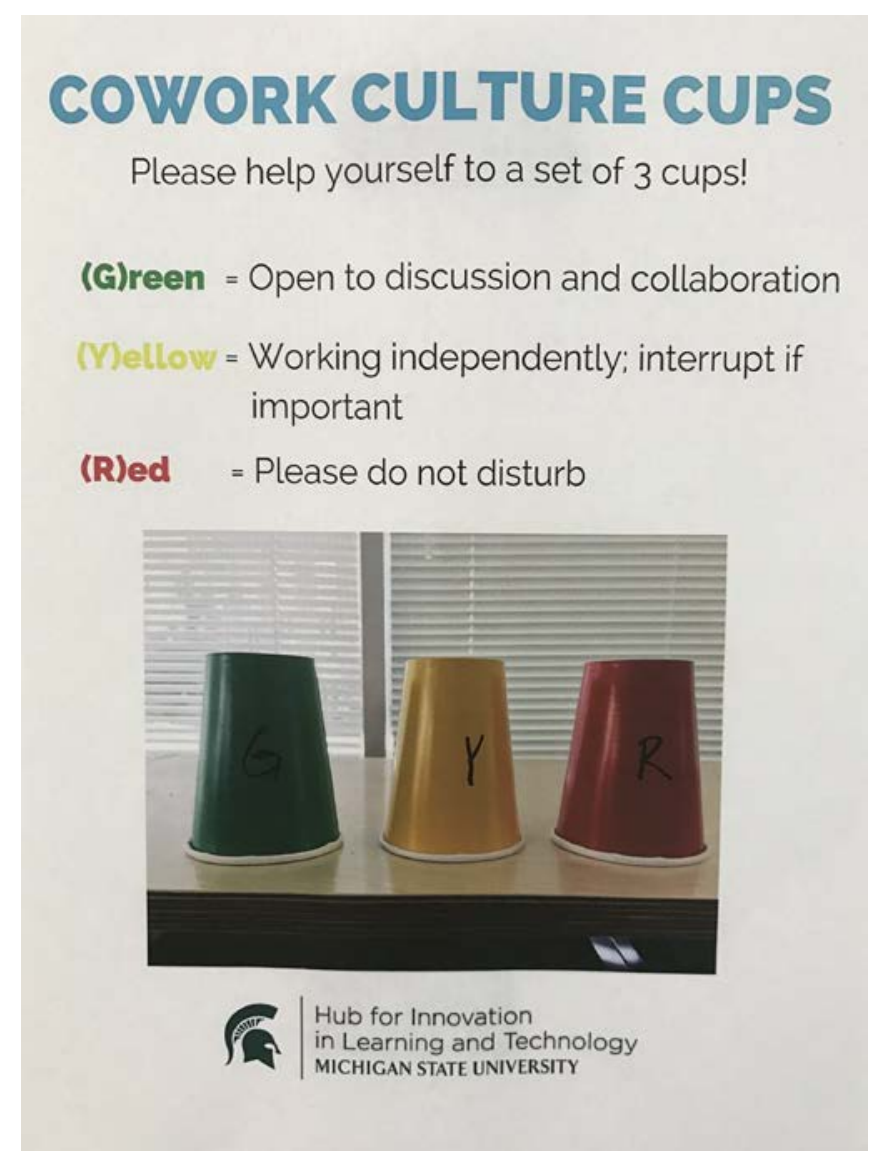

Figure 2. An internal graphic illustrating the culture cup system.

Our open workspace allows for the development of interdisciplinary networks, more collaborations, and idea generation throughout the lifespan of our projects. By working in an open space, we aim to encourage new ideas and organic conversations. We are experiencing instances where people who would not have connected on a project overhear work that parallels their own and share ideas and information. Partners across campus come to the Hub to work for the day in a new environment next to new people, expanding the opportunities for these conversations. 
Brainstorming. Brainstorming follows a similar format to coworking in that it utilizes the number of people and ideas in the room. However, while coworking is tied to co-location, brainstorm sessions are more facilitated. Individuals trying to solve problems, develop solutions, or create opportunities can come generate and discuss ideas with our team, resulting in a diverse variety of design ideas, assessment questions, and future directions.

Weekly schedule rhythms. We have developed a pattern of weekly schedule rhythms (see Figure 3 ) in an effort to create efficient workflows in a fast-paced, multi-location environment. This pattern allows our team to be more purposeful in our planning and workflow by eliminating unnecessary meetings and encouraging a cooperative culture. The Hub reserves Monday mornings for weekly project planning, collaboration, and a 30-minute standup status meeting, which we called SCRUM, and more recently, as our weekly report. Frequently seen in the software development industry, SCRUM meetings are fast-paced stand-up meetings that allow for rapid project updates and conversation on a regular basis. Individual projects still use SCRUM processes when appropriate.

Wednesday mornings are reserved for administrative meetings such as staff meeting, individual supervisory meetings, et cetera. The middle of every Thursday is "be available" time, where all Hub staff are expected to be available to contribute to brainstorm sessions and work needing additional resources. This availability allows for individuals who need more project resources to tap the collective group for formal and informal brainstorming sessions. Additionally, we ask staff to be co-located if possible during this time to allow for organic conversations and contributions to others' work.

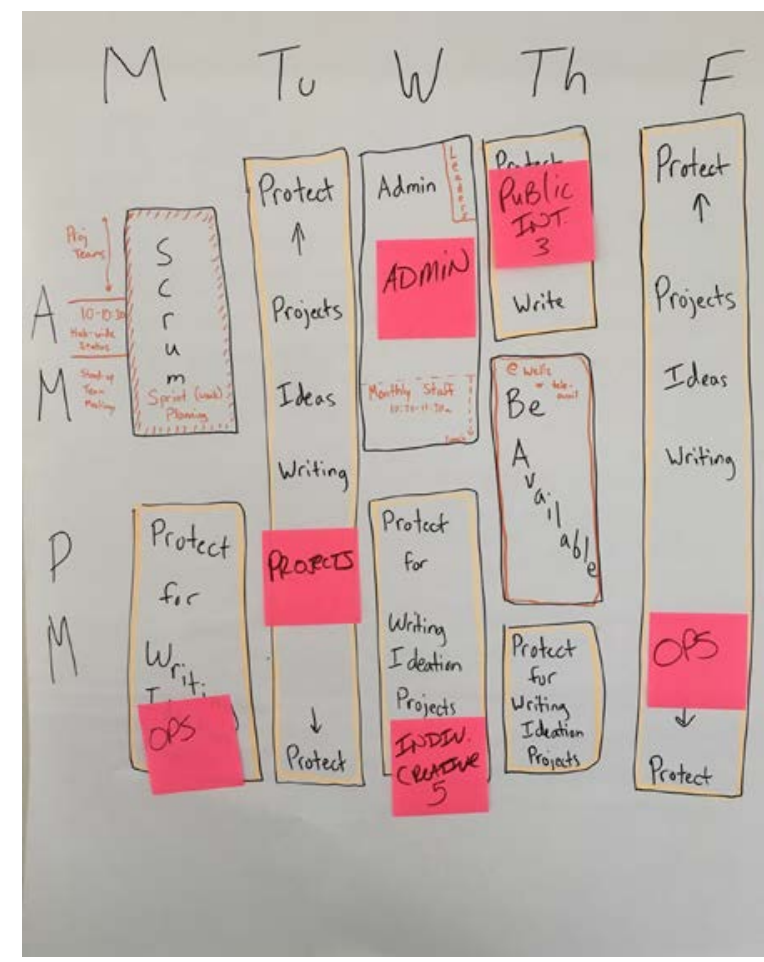

Figure 3. A graphic detailing the general weekly schedule rhythms. 
In an effort to make our projects and weekly rhythms more transparent across campus, we also invite others to attend our SCRUM meetings. We encourage our project partners to follow a similar pattern and invite them to be a part of our standing SCRUM meetings or find a block of time that works to conduct a similar status update meeting.

Agile project management. A common critique of higher education is that departments operate in isolation. While faculty publish to their scholarly communities, they often lack active sharing of good ideas and practices with each other. For example, if an academic department has a course in which many students do not succeed, then they unintentionally affect the goal of graduation, perhaps disproportionately. Without a window into that department's practices, system-level administrators or leaders are limited in their capacity to intervene. In contrast to some similar historical patterns at MSU, the Hub's processes and projects are overtly transparent and visible. We build visible and public displays for every active project in the Hub (Figure 4). Using this approach is a blend of Agile methods and transparent work environments as modeled by Menlo Innovations in Ann Arbor, Michigan (Sheridan, 2013).

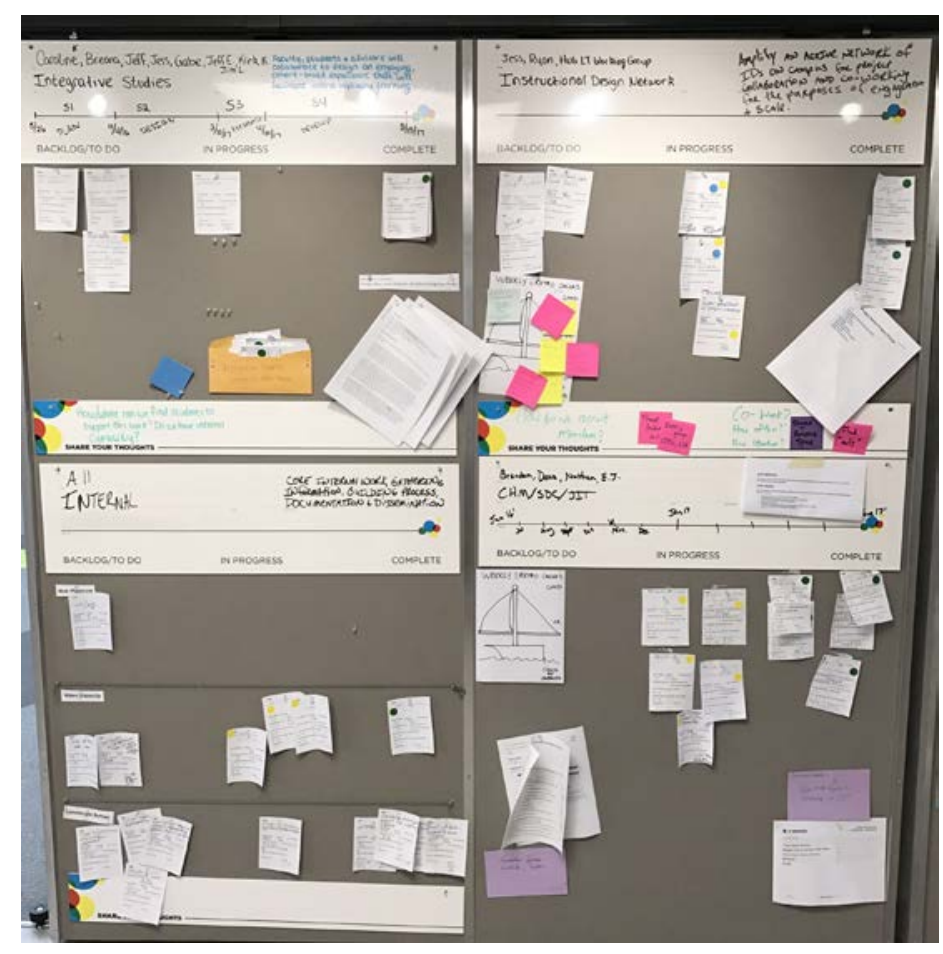

Figure 4. Projects boards on display at the Hub.

Agile project management is described as originating from a movement launched by the penning of the Manifesto for Agile Software Development in the Spring of 2001 on agileallience.org, which influenced similar movements in manufacturing, construction, aerospace and even project management itself (Highsmith, 2009). The original manifesto describes four key values as a better way of developing software: (a) individuals and interactions over processes and tools; (b) working software over comprehensive documentation; (c) customer collaboration over contract negotiation; and (d) responding to change over following a plan. 
Here, the authors of the manifesto highlight “...while there is value in the items on the right, we value the items on the left more,” (Beck et al., 2001).

Although the product of our work in the Hub is not software, we have drawn from these and other similar principles in the way we go about our work. For instance, the project boards prominently displayed in common areas used in weekly SCRUM meetings represent current progress on key project deliverables, in ways that can quickly represent pivots needed on a weekly basis. Routine operations such as meetings and professional development activities do not currently appear on these boards, but we are experimenting with ways to make notes and feedback visible and usable to others, as an expression of our goal to work transparently. This includes making notes on what is working well and what is failing.

Agile methodologies inform a unique approach to failure and the role it plays in projects. For instance, Menlo Innovations references a large and visible sign on their wall that says "Fail Faster" (Sheridan, 2013, p. 125). The concept of embracing failure seems counterintuitive, but we believe it is imperative for helping the project become more successful in the end. Where traditional project management is often put in place ultimately to avoid failure, Agile approaches intentionally seek and welcome failure as early and often as possible in the process to serve as feedback. Our approach towards the cultural value of transparency is in direct contrast to traditional examples of large-scale projects that ultimately failed out of fear of disclosing fail points earlier on in the project.

The task cards and timelines on the project boards keep us accountable to each other and our stakeholders, and serve as a physical representation of our transparent culture, progress, setbacks, successes and opportunities to learn. Engagement strategy. From the beginning, the Hub utilized a robust engagement and communications strategy to communicate the values and practices of the new unit to increasing numbers of stakeholders and interested parties. We engage our visitors and guests with visual displays and an active co-work environment. Externally, we connect with campus community members through electronic and social media outlets. We maintain our relationships with our project partners through ongoing assessment and communications. In each case, we anchor communications to several key principles, or "story anchors," summarized by the terms: Individual Strength, Collective Power, and Extraordinary Impact. Each principle is grounded in an assessment and data collection strategy to help reiterate alignment to the mission of "reinventing MSU as a learning institution."

Project pacing for external partners. We try to schedule our project work according to locally adapted Agile principles. An example of rhythm and pacing is our work on a project to redesign several general education course sequences to include linked courses, cohorts, and relevant cocurricular experiences. First, a project plan took shape consisting of phases mapped out over the course of the year with deadlines, outcomes, and deliverables. Within each phase, there are planned and focused work times, which last 1-2 weeks to maintain project momentum. Then we established patterns and rhythms with faculty on this co-curricular project by planning design sprints and regular check-in points. Faculty on this project work collaboratively with three interdisciplinary studies directors and the Hub project support team to complete these design 
sprints. Hub staff also mapped out the professional development needs for individuals on this project for the coming year.

From a project management standpoint, we enact timelines and deliverables. However, we would not consider ourselves successful if that is all we added to a project. For an external partner to work with the Hub constitutes some agreement, tacit or explicit, with trying to work differently. Our project facilitation practices regularly raise the question of project design through use and reflection, iteration, transparency, and collaboration. Materials and support resources are prepared to guide work-groups through the design sprints. To keep groups accountable, we establish regular weekly check-in meetings with agendas, minutes, and assigned tasks for each working group member to complete by the next meeting. Similar pacing behavior including planning and execution with small working groups, regular check-in meetings, agendas, and assigned tasks has seen different levels of success in a number of Hub projects.

Project staffing strategies. The Hub works to emphasize the individual strengths of experts on campus by creating and nurturing opportunities to leverage the expertise and influence of faculty and staff across our campus. In this way, we steward campus and departmental goals and share the burden of the institution to make an extraordinary impact on student experiences. MSU has an excess of intellectual capacity among experts on campus who are able and willing to help address major challenges in both pedagogy and student success and few opportunities to apply their knowledge. Our practice of engaging experts from around campus allows us to maintain a flexible staffing practice for short- and mid-length projects. Similar staffing practices have been used in higher education for many years (Bresciani et, al, 2010; Jones, Lefoe, Harvey, \& Ryland, 2012), and we find that this staffing practice attracts experts in part because people genuinely want to help solve a problem and because it enhances individual and professional networks.

Additionally, engaging people across campus for flexible staffing also helps us build human capacity in collaborative, iterative work practices that we believe will help identify new solutions to big challenges. We aim to build capacity in project management skills across campus as well, so the benefits we offer to a partner are as much a product as they are an experience with a coherent process. In turn, we commit to ongoing individual development through a linked network and through professional communications, which we align with project goals and outcomes to amplify the extraordinary work of our expert partners.

Self-reflective behaviors. In an attempt to break out of old habits and to create new capacity, we undertook the first of what would become a series of self-reflective behaviors. In January of 2016, the Hub explored the mental models held by eight individuals in the Hub leadership team. This group had been meeting weekly from October 2015 to January 2016 and intermittently beforehand to implement the vision of the Hub. A conversation recurred about the future nature of the organization, which we were still imagining. Because the group came together in October 2015 from three units on campus, each with a distinct organizational culture, a fair amount of cultural diffusion and negotiation was underway by January of 2016. At that time, the Hub's Director of Assessment led a small assessment project with the help of two research assistants. The inquiry team examined mental models as a basis for action, similar to habits of mind. An essential function of this research was to identify how this team was trying to fit into or change the campus. We define mental models here as an individual person's conscious or subconscious 
conceptualization of information and experience that drives action. As articulated by Holland, Holyoak, Nisbett, \& Thagard (1986), these models are often, but not always, illustrated in the form of metaphors and frames of understanding. Mental models often take the form of declarative knowledge (knowing what), structural knowledge (connections between ideas), and procedural knowledge (knowing how to do). Enacted mental models help an individual negotiate and process information in the context of myriad background and context influences (Table 1).

Table 1. Mental models interview questions

\begin{tabular}{|l|l|}
\hline DECLARATIVE & $\begin{array}{l}\text { - Who are you (individual or group) working for? } \\
\text { - What values drive chosen projects? (What projects should be added to } \\
\text { portfolio?) } \\
\text { - What kind of structure does an individual envision? }\end{array}$ \\
\hline STRUCTURAL & $\begin{array}{l}\text { - With whom do they want to communicate? } \\
\text { - With whom do they need to communicate? } \\
\text { - What is difference between project and service? } \\
\text { PROCEDURAL }\end{array}$ \\
& $\begin{array}{l}\text { - How do projects relate to and inform each other? } \\
\text { - How do individuals work as project leaders? As a member of a team? } \\
\text { - How do we communicate? } \\
\text { - How are projects selected? } \\
\text { - In what order does communication take place? } \\
\text { - What issues of caution does the Hub encounter or anticipate? }\end{array}$ \\
\hline
\end{tabular}

The team conducted interviews and analyses consistently with an open interpretive coding practice akin to phenomenography (Rands \& Gansamer-Topf, 2016). Participants were eight members of the Hub's leadership team. The interviewer made coding and initial interpretations, along with a member of the leadership team, and a research assistant. By discussing these interpretations with the leadership team, we invited a broader effort toward shared understanding. As a new organization, the team expressed some trepidation at this early opportunity to work transparently with each other. The interviews, analyses, and visualizations helped the leadership team translate their existing organizational patterns into a shared mindset. Table 2 (below) shows themes and attendant descriptions that emerged from eight interviewees. 
Table 2. Themes from mental models interviews

\begin{tabular}{|c|c|}
\hline \multirow[t]{4}{*}{ People } & $\begin{array}{l}\text { Connecting stakeholders/customers/whoever has a need to ideas (design, know- } \\
\text { how, IT \& technology, relationships \& communication, listening) }\end{array}$ \\
\hline & $\begin{array}{l}\text { Focus on stakeholder groups (assume people are working/important within that; } \\
\text { it's not that the entire organization is dependent on specific key individuals but it's } \\
\text { how The Hub hangs together in relation to other organizations) }\end{array}$ \\
\hline & Mention internal MSU stakeholders/people \\
\hline & Mention external stakeholders (industry, non-MSU) \\
\hline \multirow[t]{4}{*}{ Ideas } & $\begin{array}{l}\text { Enhancing ongoing discussions/conversations (ideas, projects, possibilities, re- } \\
\text { thinking) }\end{array}$ \\
\hline & Gives concrete examples \\
\hline & Gives abstract framework \\
\hline & Mention dangers, problems, pitfalls \\
\hline \multirow[t]{2}{*}{ Projects } & $\begin{array}{l}\text { Multiple/big range of topics, open-ended (R\&D, degree programs, departmental } \\
\text { initiatives) }\end{array}$ \\
\hline & Projects lead to (change, innovation, new possibilities) \\
\hline \multirow[t]{5}{*}{ Structures } & The Hub of activity (ideas, projects, possibilities) \\
\hline & $\begin{array}{l}\text { Describes elements of a horizontal/flat, open-ended, fluid, flexible organizational } \\
\text { structure }\end{array}$ \\
\hline & $\begin{array}{l}\text { Describes elements of vertical hierarchy, not necessarily rigid power structure but } \\
\text { could be well-defined roles/responsibilities }\end{array}$ \\
\hline & Focus on projects as the basic structure or driver of activity \\
\hline & Focus on services as the basic structure or driver of activity \\
\hline
\end{tabular}

There was a sense that the Hub was--and perhaps still is--at the intersection of a variety of ideas, projects, and possibilities, and that part of the Hub's job was to connect people and resources together. Key resources clearly included design thinking, knowledge, process strategies, relationships, and communication. Equally clear was that the Hub did not want to be perceived solely as a technology incubator. Most people mentioned the Hub’s role in improving or enhancing ongoing discussions on a wide range of topics, and not concentrated on one specific type of project.

Participants varied in their assertion that the Hub's consulting services and partnerships were helping to advance innovative ideas (i.e., in a curriculum). Participants did not agree that the 
Hub's way of working, which was consultative and facilitative, in general would lead the campus to innovation and change. All participants mentioned stakeholders on the MSU campus such as the provost, faculty, staff and leaders, and students; several participants also mentioned external stakeholders, such as industry and markets. These comments pointed to additional opportunities if the Hub looked outside of campus for possible partnerships.

The participants, expressing the desire for a relatively "flat" organizational structure, identified the basis for the current organizational practices. A flat organizational structure, they believed, would include intentionally informal activities to lower barriers and create more transparency. The one participant who described a more traditional vertical hierarchy also described himself or herself playing multiple roles (leader, major role, or minor role) in various projects underway simultaneously. Another participant's idea of a distributed labor platform was later implemented as our project management and essential staff assignment structure. This structure, combined with efficient time and project management, provided the most flexibility for an innovative organization with a broad outlook.

While the mental models interviews were not meant to be predictive, they were illustrative of differences in opinion about the organization as embodied by the staff. Discussions allowed for processes and ideas to surface in new ways, and thus served as an important real-time, selfreflective prompt for the team. In looking at our journey, we can point to several instances where the now-current ideas emerged from the thinking and experiences of employees during the early months of discussions, trials, and errors.

\section{Feedback and Indicators}

The assessment strategy of the Hub aims to help us know more about our work in practical and informative ways. The Hub designed its approach to yield both formative and summative analyses of data, information, practices, and impacts. The strategy encompasses a collective effort among staff to listen for and recognize various indicators of success. Individual team members may need to learn how to identify or recognize such indicators across engagement, risk, human capacity, and tangible goals. Other organizations might assign such tasks to different groups such as business development and human resources. But in the Hub, projects will only be considered successful if teams attend to the whole.

\section{Engagement}

Complementing these essential project indicators and feedback is a formal external engagement and communications strategy, utilizing story anchors, and an institution-focused assessment plan with regular reporting. Story anchors serve as the connection point for Hub cultural values, project assessment data, and external facing reports and communications. Assessment reports draw on records of indicators and feedback captured in a variety of media and networks including social media, Agile project boards, internal communications, space use and calendars, and other specific reports such as the self-study mentioned earlier (Figure 5). In turn, feedback from summative reports and external engagements inform the formative assessment loop. 


\section{ASSESSMENT STRATEGY}

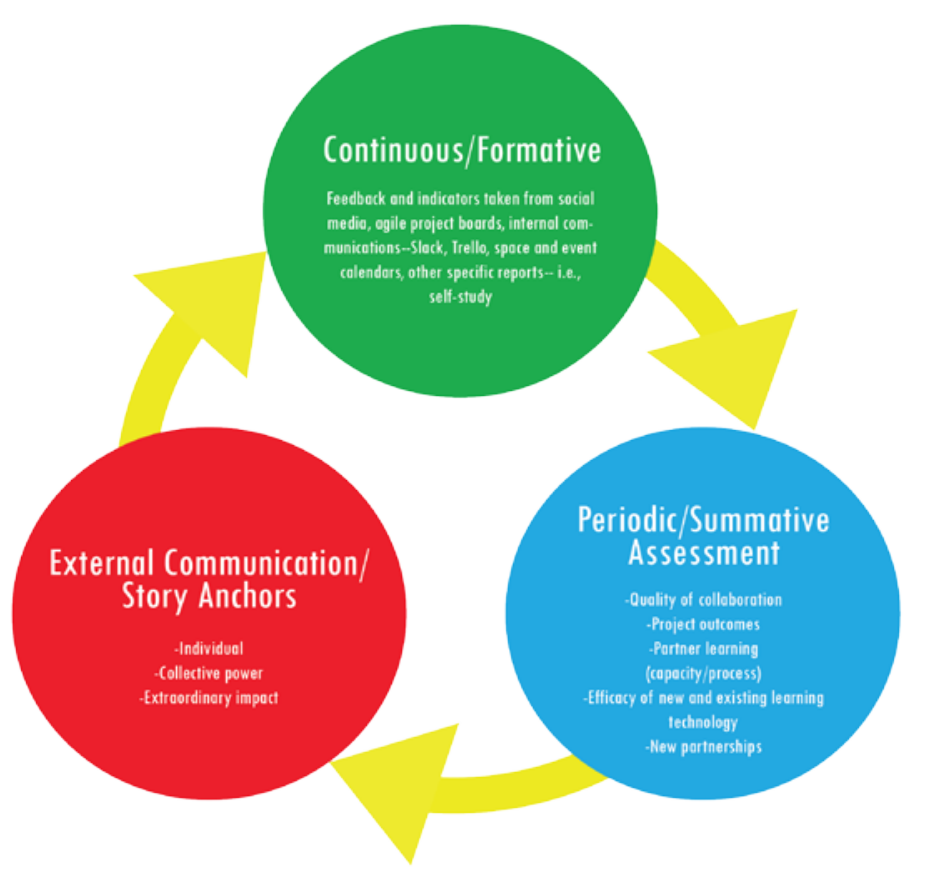

Figure 5. Assessment and Feedback Strategy.

Currently, data for the summative assessment metrics are being gathered for our first external facing report (Grabill, 2017). The use of continuous indicators, feedback, and engagement strategies drive the Hub forward. Several important indicators are described that have helped us define our work and patterns for the campus.

We track engagement for our various social media and our main website. The engagement indicators with these pages and accounts have seen more growth than anticipated, most notably with external accounts such as Facebook, which saw an increase from 0 followers in October of 2015 to 660 and counting in December of 2016. Further, the Hub website has seen a steady influx of visitors ranging from 36 to 445 per week from January to December 2016. In addition to external metrics, we also track internal communications with our team via the team-messaging platform Slack. These data then inform the ways in which we communicate and interact with our partners, and help us identify and hone strategies that are timely and effective for the collaborative culture we are trying to establish. Monitoring for project deliverables is the primary impact analyses strategy for our early efforts. As mentioned previously in the weekly time rhythms section, our team reports on project progress and deliverables each Monday morning during our reporting meeting. 


\section{Risk-Ttaking on Behalf of Others}

At times, the social-political risk of behaving differently among peers is too great (Bolman \& Deal, 2013). Because some staff and faculty on campus will not effectively change work patterns until evidence of a clear impact and reward is present, an important indicator we attend to is risk. We have taken an initial risk on behalf of improving student success by creating this organization with an open and collaborative work culture, rather than rely on somewhat isolated efforts. We took a second risk by publicly committing to goals and accountability. We collaborated internally and externally to build a set of projects that more intentionally execute designed innovations. We open our practices and work patterns to external analyses to gain insights faster. Simultaneously, the Hub aspires to be an example for others on campus to take similar risks and normalize the uncertainty associated with taking on projects with unknown outcomes. However, this kind of transparency makes it imperative that we share both successes and failures publicly. Further, as the designated "innovation" space, we must not own the projects, for our partners, but rather facilitate the success of others. To facilitate this load sharing, Hub staff share project management and planning responsibilities with project partners. We intentionally place ourselves in positions to be accountable on behalf of the project for rhythm and pacing of work, and for keeping groups on task to push projects forward.

\section{Human Capacity}

From a human and project capacity standpoint, the Hub balances between large-, medium-, and small-scale projects. Across each of these projects, there are similar characteristics of time, effort, planning, and implementation. Generally speaking, we have focused our early efforts on projects that make relatively fast, highly visible impact on initiatives in student success. Further, we have set clearer limits about the kind of projects we take on. For example, we work not to replicate services that are already available elsewhere on campus. In these situations, instead of a production role, we take more of a facilitative role, connecting needs to existing services or collaborations.

To support internal human and project capacity more efficiently, we have adopted new meeting structures. Each meeting has clearly articulated agendas, with goals that maximize productivity and reduce wasted time. We have prioritized working together through our shared scheduling rhythms, allowing for fewer and more efficient meetings. We have adopted searchable messaging and project management tools such as Slack, Trello, and Google Drive, which allow us to self-serve resources that might have otherwise required a meeting. From a technology support and service perspective, we have shifted our focus away from the manual development and production of online courses for faculty. Instead, we work with faculty and departments to teach them how to do most course production work independently, and focus more development time on development of resources requiring advanced technical skill such as immersive environments and interactive media. 
Tangible Patterns and Goals

In October of 2016, we opened a new physical space on MSU's campus that is at once an office and a public expression of our values and practices. In this new space, we have allowed for experimentation with norms for noise, communication, function, space uses, and addressing challenges based on emergent properties of the space. Since moving into the new space in a central campus location, we have been better able to observe the social systems and organizational habits of the Hub. Prior to moving during October of 2016, the Hub team worked in separate locations on campus. Efforts to make organizational and administrative patterns explicit occurred largely online or at individual meetings. Working in a central location where all Hub employees and partners can co-locate provides opportunities for patterns, habits, and other social systems to become more explicit in everyday activities and communication. We are developing a better understanding of our team members, our partners across campus, skill sets, and our capacity by working together in a central location on a regular basis. Rather than working in sequestered offices, we are now working in an open office environment, which provides daily opportunities for parallel projects to intersect in casual conversation.

Our senses are engaged in a number of ways in this open work environment. We can see physical signs of the workflow of the Hub when task cards move on team members' project boards, we can hear when project partners stop in for a meeting at the next table and when there is a buzz of discussion in the space. While the Hub has been operational since January 2016, moving into a single space in October 2016 provided tangible opportunities to track and understand our systems and work. Working together has provided the opportunity for us to gather meaningful feedback on internal software, social media, and project collaboration, each helping to inform what our next steps can or should be.

\section{Learning and Improving}

We have observed changes among Hub staff for increased individual capacity for Hub projects and identified examples of how our projects influence work patterns, pacing, and outcomes for our project partners. A discussion of these follows. We realize that an opportunity like working to reinvent MSU as a learning institution requires preparation to take risks and to fail. Innovation and change can only happen if we collectively acknowledge this risk from the outset. Early planning messages proposing the existence of the Hub pointed to indicators of success:

We will have some success by designing or engineering outcomes. But most important will be creating incentives, patterns of behavior, and supporting spaces that facilitate connections that enable the Hub and MSU to benefit from accessing information, opportunities, people, and ideas and putting those assets in relationship to each other. Innovation will be a function of the Hub's ability to connect, communicate, discover, and execute in partnership with others (Grabill, personal communication, 2015).

It is too early to determine if the Hub has yet realized the transformational goal of reinventing MSU as a learning organization. In fact, many of the active projects may take three to five years before we will be able to determine meaningful progress on the project audience. At the same time, we reflect here on how the applied ideas behind the culture building, the spatial designs, 
and our collaborative projects have influenced the daily habits of our work to be more agile, adaptive, transparent, and communicative.

\section{Lessons Learned on Spatial Design}

Previously, we discussed some of the group's experiences designing and inhabiting a new space. Although the general sentiment of employees in this new space has been relatively positive in terms of the effect it has had on complementing our cultural efforts, there have also been lessons we have learned in the time we have occupied it. As we continue to learn and work simultaneously, it is our intent to share our findings publically for other groups who may want to experiment with similar practices. For instance, the previously mentioned culture cups that indicate our individual availability status came about in response to the sometimes loud and distracting nature of the open work environment. Although in our spatial designs, we designed "focus rooms" geared for projects or meetings that need a quieter and distraction-free environment, there are times where employees of the Hub are working at their desks and need to focus on a project and indicate that it is not a good time to be interrupted. When all concerned collectively recognized this as a need, a co-work expert in the Hub adapted quickly by finding a solution to test using these color-coded culture cups. The cup system was adopted from other open working space models. In fact, part of the reason we collectively value the importance of sharing what we are learning in public spaces is because we benefit from others doing the same. We not only learn from other working groups around MSU's campus, but also continue to learn from groups at other institutions and in other sectors around the world. These groups often report similar reciprocal benefits from the practice of "learning out loud," either as public intellectuals or even as acts of benevolence from kind people willing to share what is working and what isn't.

\section{Lessons Learned on Culture}

The cultures, work rhythms, project plans, management tools, communication plans, assessment plans, and events each represent our attempt to create a culture of intentionality. We aim in turn to build capacity among our colleagues and peers for similar work, thus reinforcing our culturallevel impact on the campus. We have begun to see evidence of these impacts in the course of an academic year. In three cases, leaders from other areas of campus have asked us to help them be a hub-like operation for their subset of tasks. Further, our network building efforts have yielded new partnerships and relationships with various colleagues integrating certain behaviors. Our colleagues see examples of unexpected collisions yielding new collaboration such as hosting coworkers, technology workgroups, reputation building, and social media engagement. By celebrating these successes, we do not want to imply that these processes have been easy. Reconfiguring service offerings and learning to say "no” has been a challenge. In some cases, departments and individual faculty had been accustomed to seeing production and coursebuilding services being the primary focus of what instructional support and innovation was. Additionally, moving a group of designers and researchers with different instructional, technical, and professional philosophies into an open workspace, with new, different, and sometimes contradictory expectations and processes has been challenging. At the same time, we have aimed to help people grow as individuals, colleagues, and professionals. 


\section{Lessons Learned on Engagement with Partners}

As with any new endeavor, what matters externally includes our reputation for being a good partner. We have internally defined being a good partner by linking quality, openness, and flexibility in support work to the success of the project. Further, we aim to create ongoing, meaningful engagement opportunities and metrics for our visitors, participants, and project partners. This includes informal conversations that take place because of us working in a space intended to be open, inviting, and accessible to the broader community. It also includes existing in relatively more formalized events like our coworking sessions, meetings with external stakeholders, and project-specific design-thinking workshops. Some events are more formal than others, depending on the nature of the work but each come wrapped with intentionally collaborative planning, delivery, and reflective components. For instance, in "Eight Lessons (We Learned Yesterday) for Running a Design Thinking Workshop,” a blog post published by Dr. Leigh Graves Wolf (2016), Assistant Director of the Hub, reflects on ways we have been learning to improve our design thinking workshops. In that piece, Graves Wolf mentions things that have worked in these sessions, but also things that have not been working and what we have been doing to improve these learning experiences for our partners.

The Hub staff has conscientiously observed its own patterns for opportunities to improve, which in turn have led to an ample number of experiences and reflections. By scheduling discussion and reflection conversations - on both project work and internal processes - at regular intervals, the Hub staff have collectively created data and meaning that we believe should lead to organizational improvements. Further, by protecting time and expecting staff to contribute, the reflective work is shared. Moreover, because the culture of the Hub aims to be inclusive, the lessons reach across teams, leaders, and students in the large staff group.

\section{Conclusion}

As this small organization passes its first anniversary of practice, we find ourselves less in startup mode, with fewer experiments and more evidence of process continuity. Increasingly, a focus on project impact is becoming a central part of planning conversations in the Hub. We know that scaling up good projects is important. We also know that, to us, a good project includes content goals, capacity goals, and process goals.

We have seen that the potential value of the Hub seems to resonate with people. In some cases, they see themselves adopting some of our practices for their own organizations. To that end, we feel like we have a responsibility to share the story of the Hub. We are trying to change MSU from the inside out by being the Hub, by embracing our beliefs and culture, and encouraging other people to similarly change their institutions. MSU is not the only institution to attempt reinventing itself. We see interest elsewhere and, as the Hub, want to be a model for change. We want to see a cultural revolution within higher learning institutions, and believe that the best way to achieve this is for others to adopt similar practices of work and reflection. We think this diffusion of practices and ideology will result in a movement toward collaborative, effective learning organizations within higher education. As the Hub continues in its experiment, we see excitement, interest, confusion, and debate around the concepts of the Hub. We are regularly asked: Can this work? We respond with a simple transformation: How can this work? 


\section{References}

Aronson, D. (1996). Overview of systems thinking. Retrieved from http://resources21.org/cl/files/project264_5674/OverviewSTarticle.pdf

Argyris, C. \& Schön, D.A. (1996). Organizational learning II. Reading, MA: Addison-Wesley.

Beck, K., Beedle, M., van Bennekum, A., Cockburn, A., Cunningham, W., Fowler, M.,...Sutherland, J. (2001). The Agile Manifesto. Retrieved from https://www.agilealliance.org/agile101/the-agile-manifesto/

Bolman, L. G., \& Deal, T. E. (2013). Reframing organizations: Artistry, choice, and leadership ( ${ }^{\text {th }}$ ed.). San Francisco, CA: Jossey-Bass.

Bresciani, M. J., Todd, D. K., Carpenter, S., Janosik, S., Komives, S., Love, P., \& Tyrell, S. (2010). ACPA/NASPA professional competency areas for student affairs practitioners. Washington, DC: American College Personnel Association, National Association of Student Personnel Administrators.

Brown, T. (2008, June 1). Design Thinking. Harvard Business Review, 86(6), 84-92. Retrieved from https://hbr.org/2008/06/design-thinking

Cranton, P., \& Carusetta, E. (2004). Perspectives on Authenticity in Teaching. Adult Education Quarterly, 55(1), 5-22. http://doi.org/10.1177/0741713604268894

Fulmer, R. M. \& Keys, J. B. (1998). A Conversation with Peter Senge: New Developments in Organizational Learning. Organizational Dynamics, 27(2), 33-42. https://doi.org/10.1016/S0090-2616(98)90022-5

Grabill, J. (2017). Annual Report. Retrieved from https://hub.msu.edu/report2017/

Graves Wolf, L. (2016, August 18). Eight Lessons (We Learned Yesterday) for Running a Design Thinking Workshop [Blog post]. Retrieved from http://hub.msu.edu/eight-lessons-welearned-yesterday-for-running-a-design-thinking-workshop/

Highsmith, J. (2009). Agile project management: creating innovative products. Boston, MA: Pearson Education, Inc.

Holland, J. H., Holyoak, K. J., Nisbett, R.E., \& Thagard, P. R. (1986). Induction: Processes of inference, learning, and discovery. Cambridge, MA: MIT Press.

Jones, S., Lefoe, G., Harvey, M., \& Ryland, K. (2012). Distributed leadership: a collaborative framework for academics, executives and professionals in higher education. Journal of Higher Education Policy and Management, 34 (1), 67-78.

https://doi.org/10.1080/1360080X.2012.642334 
Kreber, C. (2004). An analysis of two models of reflection and their implications for educational development. International Journal for Academic Development, 9(1), 29-49.

https://doi.org/10.1080/1360144042000296044

Kuhn, T. (1962). The Structure of Scientific Revolutions. Chicago, IL: The University of Chicago Press.

Liimatainen, L., Poskiparta, M., Karhila, P., \& Sjögren, A. (2001). The development of reflective learning in the context of health counselling and health promotion during nurse education.

Journal of Advanced Nursing, 34(5), 648-658. https://doi.org/10.1046/j.1365-

2648.2001.01794.X

Mezirow, J. (1997). Transformative learning: Theory to practice. New Directions for Adult and Continuing Education, 1997(74), 5-12. https://doi.org/10.1002/ace.7401

Rands, M., \& Gansemer-Topf, A. M. (2016). Phenomenography: A methodological approach for assessment in student affairs. Journal of Student Affairs Inquiry, 1(2), 1-22.

Senge, P. M. (1996). Leading learning organizations. Training \& Development, 50(12), 36-37.

Senge, P. M. (2006). The fifth discipline: The art and practice of the learning organization. New York, NY: Currency: Doubleday.

Sheridan, R. (2013). Joy, Inc.: How we built a workplace people love. New York, New York: Penguin Group.

Taylor, E. W. (1997). Building Upon the Theoretical Debate: A Critical Review of the Empirical Studies of Mezirow's Transformative Learning Theory. Adult Education Quarterly, (48)1, 3459. https://doi.org/10.1177/074171369704800104

Turnali, K. (2013, April 16). What is design thinking? [Blog post]. Retrieved from http://www.digitalistmag.com/innovation/2013/04/16/what-is-design-thinking-030869 


\section{Author Information}

Bill Heinrich is the Director of Assessment in the Hub at MSU focused on assessing things that are hard to assess. He earned a Ph.D. In Higher, Adult, and Lifelong Education from Michigan State University with scholarship centered on outcomes and impacts of experiential learning and the mindsets that individuals carry about assessment work. Bill's background in student affairs and outdoor education help him translate research into practice for greater impact on institutional cultures and practices.

*Bill Heinrich, PhD

Director of Assessment, MSU Hub for Innovation in Learning and Technology

Michigan State University

D-101 Wells Hall

619 Red Cedar Rd.

East Lansing, MI 48823

Email: heinri19@msu.edu

Telephone: 517-432-6738

Twitter: @billhridesbikes

Breana Yaklin is a Learning Experience Designer for the MSU Hub for Innovation in Learning and Technology and for Teaching and Learning Technology with IT Services. She supports faculty to design strong student-focused learning experiences, and has been conducting interviews with undergraduate students to gather student voice and better inform curriculum design. Lately, she has been working closely with academic advising units to support proactive advising and student success change initiatives.

Breana Yaklin

Learning Experience Designer

Hub for Innovation in Learning and Technology

Michigan State University

619 Red Cedar Rd

D101 Wells Hall

East Lansing, MI 48823

Email: yaklinbr@msu.edu

Telephone: (517) 353-2993

Twitter: @BreanaYaklin 
David J. Goodrich is an educator and learning designer who began working at Michigan State University in 2012. Dave works in The Hub for Innovation for Learning \& Technology where he collaborates with faculty to develop new courses, redesign existing courses, and consult on instructional learning object projects. He also enjoys facilitating faculty workshops and being a guest instructor from time to time for MSU's College of Education.

David J. Goodrich

Learning Experience Designer

Hub for Innovation in Learning \& Technology

Michigan State University

619 Red Cedar Rd

D101 Wells Hall

East Lansing, MI 48823

Email: daveg@msu.edu

Telephone: (517) 884.0669

Twitter: @rangerdavie

Jessica L. Knott is the Learning Design Manager for MSU Information Technology and the MSU Hub for Innovation in Learning and Technology. She holds a Ph.D. In Higher, Adult, and Lifelong Education from Michigan State University. Her research interests include the effect of human-centered design and Agile processes in higher education, workforce development, and the impact of cross-institutional collaborations on staff and student experiences.

Jessica L. Knott

Hub for Innovation in Learning \& Technology

Michigan State University

287 Delta Court

East Lansing, MI, 48824

Email: jlknott@msu.edu

Telephone: 517-884-0674

Twitter: @jlknott

${ }^{*}$ Corresponding author 